\title{
CARACTERÍSTICAS SENSORIAIS DA CARNE DE CORDEIROS DA RAÇA SANTA INESS SUBMETIDOS A DIFERENTES DIETAS
}

\author{
Sensory characteristics of meat of Santa Inês lamb submitted to different diets \\ Sibelli Passini Barbosa Ferrão ${ }^{1}$, Maria Cristina Bressan², Rodrigo Palomo de Oliveira ${ }^{3}$, \\ Juan Ramón Olalquiaga Pérez ${ }^{4}$, Érika Cristina Rodrigues ${ }^{5}$, Denismar Alves Nogueira ${ }^{6}$
}

\begin{abstract}
RESUMO
As características sensoriais da carne, como sabor e aroma, podem ser influenciadas por diversos fatores, sendo que a alimentação é considerada o de maior importância. Objetivou-se avaliar o efeito de diferentes dietas sobre as propriedades sensoriais da carne de cordeiros, três dietas à base de silagem de cana-de-açúcar e polpa de citrus, em diferentes relações concentrado: volumoso (100:0; 75:25; 50:50), foram fornecidas a 21 cordeiros, machos, da raça Santa Inês, que foram abatidos aos $35 \mathrm{~kg}$, com idade média de 199 dias. Após o abate, as carcaças foram mantidas resfriadas a $-2^{\circ} \mathrm{C} / 24 \mathrm{~h}$ e em seguida retirado o músculo Longissimus dorsi (LD), para a análise sensorial. As amostras cozidas foram servidas a um painel de 12 provadores treinados para avaliação sensorial dos atributos de aparência, sabor, aroma, maciez e suculência. A avaliação realizada pelos provadores demonstrou que não houve efeito significativo $(\mathrm{P}>0,05)$ da dieta sobre os atributos de sabor, maciez e suculência. Entretanto, a aparência e o aroma sofreram influência, com diferença significativa entre os tratamentos 100:0 e 50:50. O aroma mais forte a "ovino" e a aparência mais "desagradável" foram mais evidentes nos animais alimentados com a dieta 100:0. As amostras provenientes dos animais alimentados com a dieta 50:50 apresentaram-se de melhor qualidade sensorial em relação às demais dietas.
\end{abstract}

Termos para indexação: Análise sensorial, qualidade, cordeiro, alimentação.

\section{ABSTRACT}

The sensory characteristics of meat, such as flavor and aroma, may be influenced by several factors, and the use as food is considered the most important. With the aim of evaluating the effects of different diets on the sensorial properties of the meat of lambs, three diets having sugar cane ensilage and pulp of citrus as a base, differing only in the proportion of concentrated: forage (100:0; 75:25; 50:50), were offered to 21 lambs of Santa Inês breed, that were slaughtered with $35 \mathrm{~kg}$, with an average age of 199 days. After slaughter, the carcasses were kept refrigerated at $-2^{\circ} \mathrm{C} / 24 \mathrm{~h}$ then the longissimus dorsi (LD) muscle was removed for the sensorial analysis. The cooked samples were served to a panel of 12 judges trained for sensorial evaluation of the attributes of appearance, taste, flavor, juiciness and succulence. The evaluation made by the judges demonstrated that there was not significant effects $(\mathrm{P}>0.05)$ of the diet on the attributes of taste, tenderness and juiciness. However, the appearance and the flavor were influenced, with significant difference between treatments 100:0 and 50:50. The strongest "sheep" flavor and the most "distasteful" appearance were evident in the meat of animals fed with diet 100:0. The samples proceeding from the animals fed with 50:50 diet presented better sensorial quality in comparision to the other diets.

Index terms: Sensory analysis, quality, lamb, feeding.

\section{(Recebido em 6 de junho de 2006 e aprovado em 31 de janeiro de 2008)}

\section{INTRODUÇÃO}

No Brasil, apesar do crescente aumento da ovinocultura de corte, o mercado consumidor de carne ovina ainda é bastante reduzido, uma vez que há uma oferta inconstante do produto, sem padronização e com excesso de gordura nas carcaças. Dessa forma, a produção de carne ovina vem suprindo apenas uma pequena parte do consumo interno, onde o cordeiro é a categoria de maior demanda (PILAR et al., 2002).

Zapata et al. (2000) observam que o baixo consumo da carne ovina pode estar associado, em parte, às

\footnotetext{
${ }^{1}$ Médica Veterinária, Doutora em Ciência dos Alimentos, Professora Adjunto - Departamento de Tecnologia Rural e Animal/DTRA - Universidade Estadual do Sudoeste da Bahia/UESB - Rua Paulo Hagge, 02 - Morumbi - 45700-000 - Itapetinga, BA - sibpass@yahoo.com.br

${ }^{2}$ Médica Veterinária, Ph.D., Professora - Unidade de Recursos Genéticos, Reprodução e Melhoramento Animal - Instituto Nacional de Recursos Biológicos/INRB - Fonte Boa - 2005-048 - Vale de Santarém, Portugal - mcbressan1@hotmail.com

${ }^{3}$ Zootecnista, Doutor em Zootecnia, Professor - Escola Agrotécnica Federal de Inconfidentes/EAFI - Praça Tiradentes, 416 - Centro - $37576-000$ Inconfidentes, MG - rodpalomo@yahoo.com.br

${ }^{4}$ Zootecnista, Doutor, Professor - Departamento de Zootecnia/DZO - Universidade Federal de Lavras/UFLA - Cx. P. 3037 - $37200-000$ - Lavras, MG jperez@ufla.br

${ }^{5}$ Bióloga, Doutoranda em Ciência dos Alimentos - Departamento de Ciência dos Alimentos/DCA - Universidade Federal de Lavras/UFLA - Cx. P. 3037 37200-000 - Lavras, MG - gikabio@yahoo.com.br

${ }^{6}$ Zootecnista, Doutor em Estatística e Experimentação Agropecuária, Professor - Faculdade de Ciências Médicas - Universidade José do Rosário Vellano/UNIFENAS - Campus Universitário - Rodovia MG 179, Km 0 - Centro - 37130-100 - Alfenas, MG - denismar.nogueira@unifenas.br
} 
características sensoriais, algumas vezes consideradas desagradáveis, como odor e sabor ativos e também ao baixo padrão de qualidade nas operações de abate, armazenamento e comercialização.

O sabor e odor da carne de ovinos são freqüentemente mencionados como o motivo do baixo consumo dessa carne. A exata causa para o chamado "flavour" indesejável ainda não está bem definido, mas acredita-se que a alta proporção de ácidos graxos saturados, além da degradação e reações de compostos solúveis em água, durante o cozimento, sejam os principais responsáveis (MELTON, 1990; VESELY, 1973).

No entanto, Sañudo et al. (1998) relatam que os fatores de aceitabilidade e as preferências específicas por distintos tipos de carcaças e carnes podem variar entre os consumidores de diferentes países e regiões. Em alguns países como, por exemplo, na Espanha, o sabor e odor característicos da carne de ovinos são apreciados e representam cerca de $53 \%$ das razões que levam à compra do produto, seguidos da maciez e suculência (13\%).

As características sensoriais da carne estão relacionadas com maciez, suculência, sabor e aroma do produto cozido. Essas características podem ser influenciadas por fatores intrínsecos como idade, sexo, raça e $\mathrm{pH}$ final do músculo, e por fatores extrínsecos como tecnologias pós-abate, tipo de cozimento e sistema de alimentação, que é considerado um dos fatores de variação de maior importância, exercendo efeito significativo sobre o aroma e o sabor da carne. A textura e a maciez são secundárias ao sabor e aroma no que diz respeito à aceitabilidade ou não da carne de ovinos e, geralmente, não constituem o principal problema. Normalmente, a carne de animais jovens e de fêmeas é mais macia que a de animais velhos e machos (BERIAIN et al., 2000; JAMORA \& RHEE, 1998; MELTON, 1990; ROUSSET-AKRIM et al., 1997).

Segundo Young et al. (1994), as características sensoriais da carne de ovinos são difíceis de avaliar objetivamente. Assim, os parâmetros indicadores de qualidade organoléptica são geralmente verificados por meio de um painel sensorial, que utiliza questionamentos apropriados, e que pode ser utilizado posteriormente para complementar o entendimento dos critérios de qualidade utilizados pelos consumidores.

Objetivou-se, neste trabalho, avaliar o efeito de diferentes dietas sobre as propriedades sensoriais dos músculos longissimus dorsi, de cordeiros da raça Santa Inês.

\section{MATERIALE MÉTODOS}

Foram utilizados 21 cordeiros machos, da raça Santa Inês, provenientes de ovelhas do rebanho do Setor de
Ovinocultura do Departamento de Zooctenia da Universidade Federal de Lavras/MG. Quando os animais alcançaram o peso de $15 \mathrm{~kg}$, com idade média de 60 dias, foram desmamados e distribuídos, aleatoriamente, em 3 grupos, recebendo dietas experimentais iso-protéicas, diferenciando apenas em seus níveis de concentrado:volumoso (tratamento 100:0 - dieta contendo $100 \%$ concentrado; tratamento $75: 25$ - dieta contendo $75 \%$ de concentrado e $25 \%$ de volumoso; tratamento 50:50 dieta contendo $50 \%$ de concentrado e de $50 \%$ de volumoso). As dietas, compostas por silagem de cana-deaçúcar e/ou polpa de citrus, farelo de soja (Glicine max L.), uréia e suplemento mineral e vitamínico foram balanceadas para atender às exigências nutricionais para um ganho médio de $250 \mathrm{~g} /$ dia, segundo as recomendações do ARC (1980). A alimentação foi fornecida duas vezes ao dia, $40 \%$ de manhã e $60 \%$ à tarde, em quantidades que permitiam uma sobra de $10 \%$ do total oferecido.

Ao atingirem $35 \mathrm{~kg}$ de peso vivo, com idade média de 199 dias, os animais foram abatidos e após resfriamento por 24 horas à temperatura de $-2^{\circ} \mathrm{C}$, da $1 / 2$ carcaça direita fria foi removido o músculo longissimus dorsi (LD), que foi embalado em papel filme (polietileno) e papel alumínio, identificado, colocado em saco plástico e congelado a $18^{\circ} \mathrm{C}$, para posterior análise sensorial. As amostras, no momento da análise, foram descongeladas a $5^{\circ} \mathrm{C}$ por 24horas. Em seguida, os músculos foram envolvidos em papel alumínio e levados ao forno elétrico a $170^{\circ} \mathrm{C}$, até atingirem a temperatura interna de $75^{\circ} \mathrm{C}$. A temperatura interna foi determinada com auxílio de um termômetro, que foi inserido no centro geométrico de cada peça. Após as peças estarem assadas, foram cortadas transversalmente em cubos de $1 \mathrm{~cm}^{3}$, e esses transferidos para um béquer pré-aquecido, codificado e coberto com vidro-relógio, permanecendo à temperatura de $45-50^{\circ} \mathrm{C}$, não ultrapassando o tempo de 15 minutos do corte, até o momento de serem servidos (FAHMY et al., 1992). As amostras já preparadas foram servidas a um painel de 12 provadores treinados, composto por 4 homens e 8 mulheres, com idade entre 25 e 45 anos, todos funcionários e estudantes de Pós-Graduação do Departamento de Ciência de Alimentos da Universidade Federal de Lavras. Os provadores avaliaram sensorialmente os atributos de aparência, sabor, aroma, maciez e suculência das amostras, utilizando-se uma escala não estruturada de $10 \mathrm{~cm}$ (FERREIRA, 2000), com os extremos menos favorável e mais favorável, com o objetivo de verificar se existiam diferenças entre os tratamentos. $\mathrm{O}$ trabalho foi conduzido no Laboratório de Análise Sensorial do Departamento de Ciência dos Alimentos - UFLA. 
O teste baseou-se em apresentar aos provadores, que se encontravam em cabines individuais iluminadas com luz branca, simultaneamente três amostras diferentes. As amostras foram servidas em recipientes descartáveis de cor branca, dotados de suportes codificados com três dígitos numéricos. Os provadores receberam as amostras e a ficha para avaliar os atributos. Após seleção e treinamento prévio dos provadores, foram realizadas 10 sessões para avaliação das amostras, com duas repetições: uma no período da manhã e outra no período da tarde. Para remoção do sabor residual da boca foi utilizada água à temperatura ambiente.

A interpretação dos resultados foi realizada efetuando-se primeiramente uma transformação do ponto marcado para uma nota. Com auxílio de uma régua, mediu-se o ponto marcado e a sua medida em centímetros foi considerada como a nota conferida pelo provador. Notas mais elevadas se aproximavam do extremo máximo (dez) e indicavam carnes com aparência mais agradável, aroma característico mais suave, sabor característico mais suave, carne mais macia e com maior suculência. Notas mais baixas se aproximavam do extremo mínimo (zero) e indicavam carnes com aparência mais desagradável, aroma característico mais forte, sabor característico mais forte, carne mais dura e com menor suculência.

Os lipídeos totais dos músculos longissimus dorsi foram determinados pelo método de Soxhlet, baseado na solubilização de lipídios (HORWITZ, 1990).

$\mathrm{O}$ delineamento experimental foi inteiramente casualizado, em que foram comparadas três dietas distintas fornecidas a ovinos da raça Santa Inês. A unidade experimental foi composta por um animal. Os dados obtidos foram submetidos ao modelo Proc Mixed do pacote estatístico SAS (SAS INSTITUTE, 1996). A análise de variância foi submetida ao teste de Tukey e identificada quando apresentou nível de significância de 5\%. O modelo experimental utilizado foi:

$$
Y_{i j}=\mu+M_{i}+E_{i}
$$

onde:

$\mathrm{Y}_{\mathrm{ij}}=$ observação no músculo dos cordeiros abatidos do tratamento $\mathrm{i}$, na repetição $\mathrm{j}$;

$\mu=$ média geral do experimento;

$\mathrm{M}_{\mathrm{i}}=$ efeito do tratamento, sendo $\mathrm{i}=1,2,3$;

$\mathrm{E}_{\mathrm{i}}=$ erro experimental associado à observação $\mathrm{Y}_{\mathrm{i}}$, que por hipótese tem distribuição normal, com média 0 e variância $\sigma^{2}$

\section{RESULTADOS E DISCUSSÃO}

Os resultados obtidos pela avaliação sensorial em relação às características de aparência, aroma, sabor, maciez e suculência estão apresentados na Tabela 1.

A avaliação realizada pelos provadores do painel sensorial demonstrou que não houve efeito da dieta sobre os atributos de sabor, maciez e suculência. Com relação à aparência e ao aroma, o painel não identificou diferenças entre os tratamentos de 100:0 e 75:25 e entre 75:25 e 50:50, mas diferenças significativas foram detectadas entre os tratamentos 100:0 e 50:50 $(\mathrm{P}<0,05)$.

$\mathrm{O}$ aroma, que pode ser entendido como aroma característico da carne de ovino, ou aroma a "ovino", foi mais evidente nos animais alimentados com a dieta de 100:0, bem como uma aparência mais "desagradável". Apesar de não serem observadas diferenças para todos os atributos, de maneira geral, a dieta de 50:50 apresentou as notas mais elevadas para todas as características avaliadas, indicando que, em uma análise subjetiva, as amostras provenientes dessa dieta teriam uma qualidade sensorial superior em relação às demais.

Para carne de cordeiro, os termos sabor e aroma a “ovino", usualmente empregados, referem-se ao sabor e aroma característicos que essa carne apresenta, independente da idade, o que é considerado, por muitos consumidores, como indesejável. Essas características tornam-se mais pronunciadas quando a carne é submetida a temperaturas mais altas de cozimento, e especialmente quando reações de escurecimento acontecem (CRAMER, 1983). Considerando-se o aroma característico, as amostras provenientes do tratamento 100:0 poderiam ser rejeitadas pelos consumidores em função das notas mais baixas que lhe foram atribuídas, caracterizando carnes com aromas mais pronunciados.

Segundo Siqueira et al. (2002), a alimentação é preponderante na determinação dos caracteres sensoriais da carne, e o uso de concentrado na dieta promove o aumento da suculência e, pelo fato de alterarem a composição em ácidos graxos da gordura, permitem modificar o sabor e o odor. Fisher et al. (2000) citam que a carne de ovinos pode adquirir características únicas de "flavour" em função da dieta fornecida aos animais, e que as diferenças percebidas em um painel são, em grande parte, resultados da variação do teor de gordura e da composição em ácidos graxos da mesma.

Em geral, algumas dietas podem alterar a composição da gordura e, conseqüentemente, alterar o sabor da carne. Dietas à base de silagem podem levar a um sabor e aroma de "suíno", quando comparadas com animais que foram alimentados a pasto (FIELD et al., 1983). 
Tabela 1 - Médias dos valores atribuídos aos atributos de qualidade da avaliação sensorial ( \pm DP) de carne oriunda do músculo longissimus dorsi de cordeiros Santa Inês, alimentados com diferentes dietas.

\begin{tabular}{lccc}
\hline Atributos* & \multicolumn{3}{c}{ Dietas (Concentrado:Forragem) } \\
\cline { 2 - 4 } & $100: 0$ & $75: 25$ & $50: 50$ \\
\hline Aparência & $7,00^{\mathrm{A}} \pm 0,10$ & $7,35^{\mathrm{AB}} \pm 0,13$ & $7,41^{\mathrm{B}} \pm 0,14$ \\
Aroma & $6,70^{\mathrm{A}} \pm 0,09$ & $6,97^{\mathrm{AB}} \pm 0,12$ & $7,15^{\mathrm{B}} \pm 0,19$ \\
Sabor & $6,54 \pm 0,22$ & $6,87 \pm 0,23$ & $7,01 \pm 0,33$ \\
Maciez & $6,01 \pm 0,21$ & $6,24 \pm 0,22$ & $6,37 \pm 0,56$ \\
Suculência & $5,33 \pm 0,25$ & $5,33 \pm 0,25$ & $5,68 \pm 0,49$ \\
\hline
\end{tabular}

Médias seguidas de letras distintas diferem entre si pelo teste de Tukey $(\mathrm{P}<0,05)$. Nas linhas, letras maiúsculas $(\mathrm{A}, \mathrm{B})$ para as dietas.

* Os atributos de qualidade foram avaliados por meio de escala não estruturada de $10 \mathrm{~cm}$, onde os extremos correspondem: 0 menos favorável e 10 - mais favorável

No presente estudo, o teor médio de lipídeos nas amostras estudadas foi de 3,28, 2,80 e 3,06\% para as dietas 100:0, 75:25 e 50:50, respectivamente. O elevado teor de concentrado na dieta não foi capaz de promover diferença significativa para a suculência, a maciez e o sabor, mas para o aroma podemos observar que a dieta com maior proporção de concentrado (100:0) recebeu a menor nota $(6,70)$ dos provadores e pode ser considerada com maior aroma característico, coincidindo com aqueles animais que apresentaram maiores teores de gordura $(3,28 \%)$. No entanto, como não houve diferença significativa $(\mathrm{p}>0,05)$ para o teor de gordura entre as dietas, não é possível afirmar que esse parâmetro interferiu nos atributos sensoriais estudados.

Os valores médios dos atributos de qualidade do presente estudo estão próximos, mas ligeiramente superiores, indicando melhor qualidade sensorial, aos encontrados por Siqueira et al. (2002), que estudando as características sensoriais do músculo Longissimus dorsi de cordeiros abatidos com 4 pesos distintos $(28,32,36$ e $40 \mathrm{~kg}$ ) e alimentados com dieta composta por $35 \%$ de feno e $65 \%$ de concentrado, observaram que, para a raça Santa Inês, não houve diferença entre pesos para os atributos avaliados, com pontuação média de 6,9 para aparência; 6,6 para aroma; 6,6 para sabor; 5,0 para maciez e 6,1 para suculência.

No entanto, Madruga et al. (2005) encontraram, de maneira geral, resultados mais elevados que os do presente estudo. $\mathrm{Na}$ análise sensorial de diferentes pedaços da perna da carne de cordeiros Santa Inês, alimentados com dietas distintas (4 tipos de alimentos volumosos $+40 \%$ de concentrado), os autores encontraram para os atributos avaliados médias que variaram de 6,65 a 7,33 para textura; 7,17 a 7,85 para maciez,
7,05 a 7,65 para sabor, e 6,57 a 7,65 para suculência. Apenas o "odor a ovino" foi mais pronunciado, com pontuação média de 4,87 .

Tonetto et al. (2004) forneceram diferentes dietas (pastagem natural suplementada, pastagem cultivada de azevém e confinamento) a cordeiros cruzas de Texel x Ile de France, com o objetivo de observarem o efeito dos tratamentos sobre as características da carne. Um painel treinado avaliou amostras de lombo e não percebeu efeito dos tratamentos de alimentação para os parâmetros de maciez, palatabilidade e suculência, com pontuação média variando de 6,8 a 7,$61 ; 6,08$ a 7,33 e 6,55 a 6,91 , respectivamente.

Em estudo sobre as propriedades físicas e sensoriais da carne ovina no Nordeste brasileiro, Zapata et al. (2000), utilizando cruzas Somalis Brasileira x Crioula e Santa Inês x Crioula submetidas a duas dietas de feno e feno $+20 \%$ de concentrado, concluíram que não houve efeito da dieta sobre os parâmetros sensoriais avaliados nas carnes de pernil. A análise sensorial, realizada por meio de uma escala hedônica de 9 pontos, frente a um painel não treinado de 24 consumidores, indicou uma boa aceitação das carnes avaliadas, com pontuação média variando de 7,04 a 7,25.

Yu et al. (2001), compararam o efeito de dietas à base de fibras (alfafa e aveia), adicionadas de leguminosas cruas e tostadas nos atributos sensoriais do músculo biceps femoris de cordeiros (Border Leicester $\mathrm{x}$ Merino $\mathrm{x}$ Poll Dorset) e não encontraram diferenças significativas para aroma, sabor, suculência, maciez e aceitabilidade total. O painel não treinado de 16 provadores indicou que aroma e sabor fortes foram mais altos na dieta com a leguminosa tostada. 


\section{CONCLUSÕES}

O uso de diferentes dietas não foi capaz de promover diferenças significativas perceptíveis pelo painel sensorial para os parâmetros de sabor, maciez e suculência. Os atributos de aparência e aroma foram influenciados pela alimentação dos animais. O elevado teor de concentrado na dieta (100\% de concentrado) levou à ocorrência de carnes com aparência mais desagradável e aroma característico mais forte.

\section{REFERÊNCIAS BIBLIOGRÁFICAS}

AGRICULTURAL RESEARCH COUNCIL. The nutrient requeriment of farm animals. London, 1980. 351 p.

BERIAIN, M. J.; GORRAIZ, C.; HORCADA, A.; PURROY, A. Sensory quality of fresh lamb meat. In: LEDIN, I.; MORAND-FEHR, P. (Eds.). Sheep and goat nutrition: intake, digestion, quality of products and rangelands. Zaragoza: CIHEAM-IAMZ, 2000. p. 125-128.

CRAMER, D. A. Chemical compounds implicated in lamb flavor. Food Technology, Chicago, p. 249-257, May 1983.

FAHMY, M. H.; BOUCHER, J. M.; POSTE, L. M.; GRÉGOIRE, R.; BUTLER, G.; COMEAU, J. E. Feed efficiency, carcass characteristics, and sensory quality of lambs, with or without profilic ancestry, fed diets with different protein supplements. Journal of Animal Science, Champaign, v. 70, n. 5, p. 1365-1374, June 1992.

FERREIRA, V. L. P. Análise sensorial: testes discriminativos e afetivos. Campinas: SBCTA, 2000. 127 p.

FIELD, R. A.; WILLIAMS, J. C.; MILLER, G. J. The effect of diet on lamb flavor. Food Technology, Chicago, v. 37, n. 5, p. 258-263, May 1983.

FISHER, A. V.; ENSER, M.; RICHARDSON, R. I.; WOOD, J. D.; NUTE, G. R.; KURT, E.; SINCLAIR, L. A.; WILKINSON, R. G. Fatty acid composition and eating quality of lamb types derived from four diverse breed $\mathrm{x}$ production systems. Meat Science, Amsterdam, v. 55, n. 2, p. 141-147, June 2000.

HOWITZ, W. Official methods of analysis of Association of Official Analytical Chemists. 13. ed. Whashington, DC: AOAC, 1990.

JAMORA, J. J.; RHEE, K. S. The uniqueness of lambs: nutritional and sensory properties. Sheep \& Goat
Research Journal, Englewood, v. 14, n. 1, p. 53-64, Jan. 1998.

MADRUGA, M. S.; SOUSA, W. H.; ROSALES, M. D.; CUNHA, M. G. G.; RAMOS, J. L. F. Qualidade da carne de cordeiros Santa Inês terminados com diferentes dietas. Revista Brasileira de Zootecnia, Viçosa, v. 34, n. 1, p. 309315, jan./fev. 2005.

MELTON, S. L. Effects of feeds on flavor of red meat: a review. Journal of Animal Science, Champaign, v. 68, n. 12, p. 4421-4435, Dec. 1990.

PILAR, R. C.; PÉREZ, J. R. O.; SANTOS, C. L.; PEDREIRA, B. C. Considerações sobre a produção de cordeiros. Lavras: UFLA, 2002. 24 p. (Boletim agropecuário, 53).

ROUSSET-AKRIM, S.; YOUNG, O. A.; BERDAGUÉ, J. L. Diet and growth effects in panel assessment of sheepmeat odour and flavour. Meat Science, Amsterdam, v. 45, n. 2, p. 169-181, Feb. 1997.

SAÑUDO, C.; SANCHEZ, A.; ALFONSO, M. A. Small ruminant production systems and factors affecting lamb meat quality. Meat Science, Amsterdam, v. 49, p. 29-64, May 1998. Supplement 1.

SAS INSTITUTE. SAS user's guide: statistics. 5. ed. Cary, 1996. $1290 \mathrm{p}$.

SIQUEIRA, E. R.; ROÇA, R. O.; FERNANDES, S.; UEMI, A. Características sensoriais da carne de cordeiros das raças Hampshire Down, Santa Inês e mestiços Bergamácia $x$ Corriedale abatidos com quatro distintos pesos. Revista Brasileira de Zootecnia, Viçosa, v. 31, n. 3, p. 1269-1272, jun. 2002.

TONETTO, C. J.; PIRES, C. C.; MÜLLER, L.; ROCHA, M. G.; SILVA, J. H. S.; FRESCURA, R. B. M.; KIPPERT, C. J. Rendimentos de corte da carcaça, características da carne e componentes de peso vivo em cordeiros terminados em três sistemas de alimentação. Revista Brasileira de Zootecnia, Viçosa, v. 33, n. 1, p. 234-241, jan./fev. 2004.

VESELY, J. A. Fatty acids and steroids affecting flavor and aroma of meat from ram, cryptorchid, and wether lambs. Canadian Journal Animal Science, Ontario, v. 53, n. 4, p. 673-678, Dec. 1973. 
YOUNG, O. A.; REID, D. H.; SMITH, M. E.; BRAGGINS, T. J. Sheepmeat odour and flavour. In: SHAHIDI, F. (Ed.). Flavour of meat and meat products. New York: Black Academic \& Professional, 1994. p. 71-97.

YU, P.; SPRAGUE, M.; EGAN, A. R.; CASTLEMAN, G. H.; LEURY, B. J. Comparison of raw and roasted narbon beans (Vicia narbonensis) on performance and meat sensory attributes of lambs fed a roughage-based diet. Animal Feed Science and Technology, New York, v. 92, n. 1/2, p. 1-16, July 2001.

ZAPATA, J. F. F.; SEABRA, L. M. J.; NOGUEIRA, C. M.; BARROS, N. Estudo da qualidade da carne ovina do nordeste brasileiro: propriedades físicas e sensoriais. Ciência e Tecnologia de Alimentos, Campinas, v. 20, n. 2, p. 274-277, maio/ago. 2000. 